\title{
Rapid percutaneous tracheal catheterization using electrical guidance
}

\author{
Ban C. H. Tsui, MD · Jenkin Tsui
}

Received: 12 September 2011/ Accepted: 19 September 2011/Published online: 12 October 2011

(C) Canadian Anesthesiologists' Society 2011

\section{To the Editor,}

Percutaneous needle tracheostomy or cricothyroidotomy establishes emergent airway access in situations of "cannot ventilate, cannot intubate." ${ }^{1}$ Currently, most clinicians and paramedics rely on palpation of anatomical landmarks and aspiration of air to guide needle puncture and confirm needle placement into the trachea. However, landmarking through palpation can be unreliable due to variability in patient sex, size, and body composition, and true aspiration of air from the trachea is hard to interpret in many cases. Moreover, difficulties can arise during needle insertion, including inability to cannulate the airway and perforation of the posterior or lateral aspects of the tracheal wall. Multiple attempts at needle insertion may be required, heightening these risks. Consequently, the complication rate of needle tracheostomy or cricothyroidotomy in emergency situations can be as high as $40 \% .^{2}$ Given this high complication rate and the critical and time-sensitive nature of the procedure, most physicians and paramedics would benefit from a reliable, objective, and unsophisticated method to increase its speed and accuracy.

Here, we invent a novel method involving the simple presence or absence of an electrical current as a means to identify and guide needle placement into the trachea. With a grounded electrode on the body, the tip of an insulated needle maintains a closed electrical circuit when in contact with any tissue - in this case, the tracheal wall or peritracheal tissues (Fig. 1). Insertion of the tip of the insulated needle into the lumen of the air-filled trachea impedes the current, opening the electrical circuit. Based on these principles, the absence of electrical conductance could

B. C. H. Tsui, MD $(\varangle) \cdot$ J. Tsui

University of Alberta, Edmonton, AB, Canada

e-mail: btsui@ualberta.ca serve as a simple practical signal to detect insulated needle entrance into the trachea. Conveniently, most modern nerve stimulators have the ability to produce an audible and/or visual signal, enabling easy and rapid detection of the presence or absence of an electrical current.

We demonstrated this concept in both a male and a female cadaver using a 21G $64 \mathrm{~mm}$ insulated needle with an $18 \mathrm{G}$ catheter assembly (MultiSet, Pajunk, Germany) and a commonly used nerve stimulator (HNS 12, B.Braun, Germany). Preliminary results proved encouraging, with $100 \%$ success in identifying the exact moment of needle tip entrance into the trachea (multiple insertions, more than five times for each cadaver), as viewed by bronchoscopy (Fig. 2). The cadavers were in legal custody of the Division of Anatomy of the author's institution, and the procedures were performed with the permission from the Division of Anatomy and in compliance with the institutional ethical standards for the use of human material in medical education. As displayed in Fig. 2, real-time needle puncture of the tracheal wall was demonstrated with a corresponding change in current from an initial setting of $1.0 \mathrm{~mA}$ to $0.0 \mathrm{~mA}$ (pulse wideth $0.1 \mathrm{msec} ; 2 \mathrm{~Hz}$ ), and it was also indicated by an instantaneous audible switch of signal tone. Importantly, this feature also serves as a warning signal, alerting the user that the needle tip is in contact with the posterior tracheal wall. The tracheal wall is also at risk of being punctured if the needle is advanced too far or is positioned too obliquely such that the needle tip touches a lateral aspect of the tracheal wall when advanced a short distance. Therefore, this technique can both confirm initial penetration of the tracheal wall and help guide positioning of the insulated needle in the airway. Once the needlecatheter assembly has been inserted successfully into the airway lumen, the needle is withdrawn. Following this step, the clinician has the option of providing temporary 

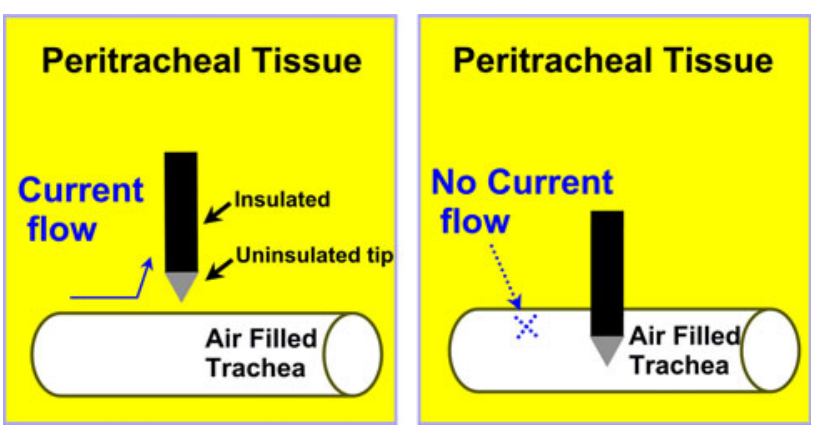

Fig. 1 Diagram of electrically-guided needle insertion. A needle situated in the peritracheal tissue (left) maintains a closed electrical circuit with current flow to the needle. The current flow stops when the needle penetrates the tracheal wall, due to the high impedance of the air-filled cavity (right)
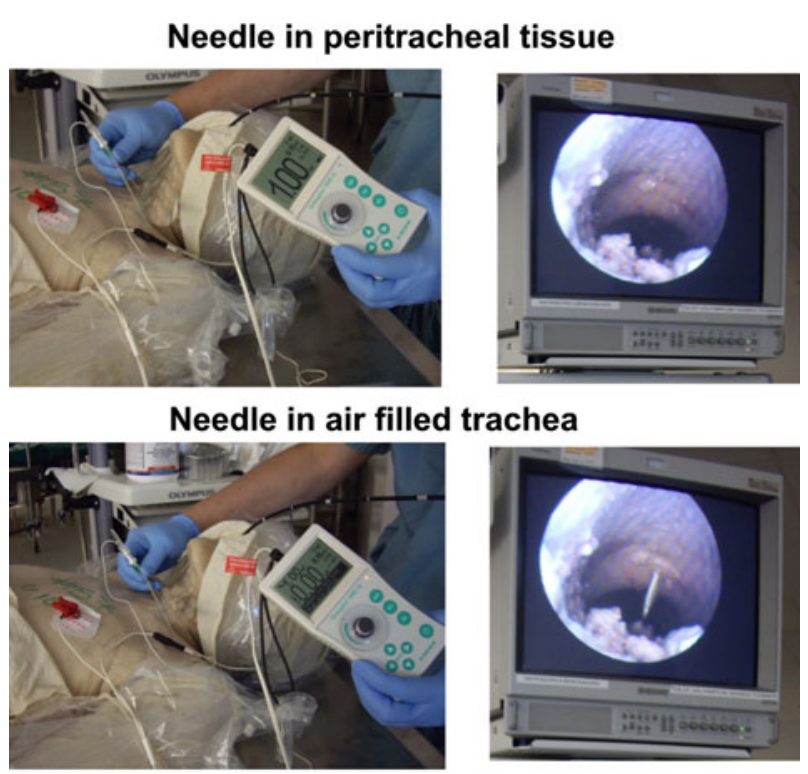

Needle touching posterior trachea
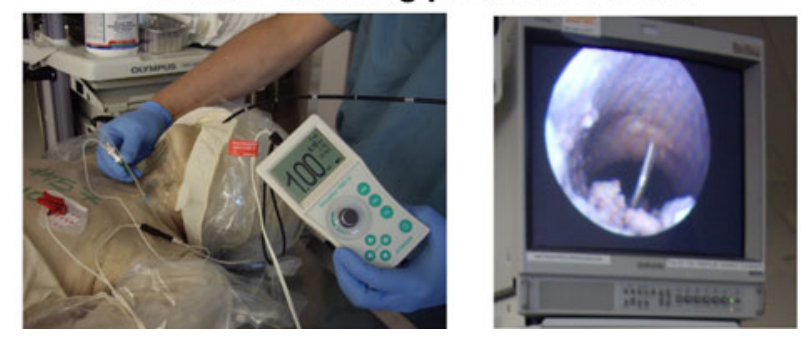

Fig. 2 Identification of tracheal wall penetration by absence of electrical current. Left panels: (top) Contact with the peritracheal tissue maintains a closed electrical circuit (1.00 mA) (HNS 12, B.Braun, Germany); (middle) Needle (MultiSet, Pajunk, Germany) penetration into the tracheal lumen impedes the closed circuit (0.00 mA); (bottom) Conductance of current is re-established upon contact with the distal/posterior tracheal wall. Bronchoscopy was used to confirm the needle position (right panels) oxygenation (e.g., jet insufflations) or establishing a longer-term ventilation system by inserting a large diameter semi-rigid tracheal cannula (e.g., cricothyroidotomy set).

Together, our preliminary observations support further evaluation of this technique as a valid method to confirm and guide needle placement into the trachea. If proven accurate and reliable, the impact of this technique will likely be significant for acute airway management, and its simplicity and ease of use should improve the success rate of the procedure. Although this technique can ensure needle placement into the air-filled space (i.e., likely trachea) in a subject with an untraumatized airway, the clinician should be aware that limited information is available with respect to the exact needle location. Therefore, locating the midline of the neck through careful palpation remains a crucial component of successful needle guidance during needle cricothyroidotomy and tracheostomy.

Acknowledgements The authors sincerely thank Jennifer Pillay and Drs. Gareth Corry, Vivian Ip, Brendan Finucane, and Anil Walji for their contributions.

Conflicts of interest None declared.

\section{References}

1. Finucane BT, Tsui BC, Santora AH. The difficult airway. In: Finucane BT, Tsui BC, Santora AH, editors. Principles of Airway Management. 4th ed. ed. NY: Springer; 2011. p. 361-414.

2. DeLaurier GA, Hawkins ML, Treat RC, Mansberger AR Jr. Acute airway management. Role of cricothyroidotomy. Am Surg 1990; 56: $12-5$ 